\title{
A very low carbohydrate ketogenic diet prevents the progression of hepatic steatosis caused by hyperglycemia in a juvenile obese mouse model
}

\author{
T Okuda and N Morita
}

\begin{abstract}
OBJECTIVE: To investigate whether the improvement in hyperglycemia by dietary control influences hyperglycemia-induced pathologies in tissues of juvenile obese $(o b / o b)$ mice.

DESIGN: Five-week-old ob/ob mice were fed a very low carbohydrate ketogenic diet (KD) for 7 weeks. The blood glucose levels and body weight were monitored during this period. Biochemical parameters in the serum and tissue pathologies of the mice were analyzed at the end of the 7-week period.

RESULTS: The hyperglycemic phenotype of the $o b / o b$ mice was improved by KD feeding for 7 weeks. Surprisingly, we found that KD feeding also drastically reduced the hepatic steatosis phenotype in ob/ob mice, while their obesity phenotype was unaltered. Sodium dodecyl sulfate-polyacrylamide gel electrophoresis analysis revealed that several proteins found in the liver of $o b / o b$ mice fed a regular chow diet were undetectable after being fed KD. Liquid chromatography with tandem mass spectrometry (LC-MS/MS) MASCOT search and western blot analysis revealed that the proteins absent from the mice fed KD included fatty acid synthase (FAS) and acetyl-CoA carboxylase 1 (ACC1), which are key enzymes for lipogenesis in the liver. Fatty acid analysis supported the results because the ratio of C18:1, which is a major product of lipogenesis, was reduced by KD feeding. However, C18:2, which cannot be synthesized in mammalian cells but is present in the KD, was found to be a major component in the liver of KD-fed ob/ob mice. CONCLUSION: Hyperglycemia promotes hepatic steatosis via the lipogenic pathway in the liver of juvenile ob/ob mice. However, the development of steatosis is prevented by feeding KD owing to an improvement in hyperglycemia. We found that the progression of steatosis is reflected by the composition of fatty acids in the total lipids of the liver and serum.
\end{abstract}

Nutrition and Diabetes (2012) 2, e50; doi:10.1038/nutd.2012.24; published online 12 November 2012

Keywords: fatty acid; hyperglycemia; ketogenic diet; ob/ob mouse; steatosis

\section{INTRODUCTION}

Metabolic diseases elicited by chronic hyperglycemia are most often associated with diabetes mellitus. In diabetic patients, serious chronic hyperglycemia causes several complications such as retinopathy, neuropathy and nephropathy. ${ }^{1,2}$ Hyperglycemia is also common in various diseases, such as vascular complications, myocardial infraction, cancer and Alzheimer's disease. ${ }^{3-6}$ Furthermore, a recent study revealed that an excessive quantity of extracellular glucose induces insulin resistance in the cell. ${ }^{7}$ This result suggests that hyperglycemia could also produce peripheral insulin resistance in vivo. However, an effective therapy for these diseases remains elusive because of a lack of information on the molecular mechanisms by which hyperglycemia induces peripheral pathologies. Therefore, a comprehensive approach to understanding hyperglycemiainduced pathologies should be systematically developed in vivo. Such an approach will provide valuable information on these complex problems, which cannot be fully addressed by cell culture-based investigations. The establishment of animal models that reflect the pathological processes is an effective research tool for understanding the developmental mechanisms of these diseases.
Recently, a unique animal model has been developed, which involves feeding a low carbohydrate ketogenic diet (KD) to obese mice $(o b / o b){ }^{8} \mathrm{KD}$ is a theoretical and nutritional balanced diet composed of high fat, adequate protein and low carbohydrate. ${ }^{9}$ The KD is generally used in seizure control in human, and recent study also proposed the effectiveness to improve other pathological processes, including renal disease ${ }^{10}$ and cardiac function. ${ }^{11}$ The KD mimics glucose starvation in the body and produces ketone bodies from fat, which supplies energy to peripheral tissues. It has been suggested that feeding $K D$ to mature $o b / o b$ mice, which exhibit obesity, leads to a change in the expression of a specific set of genes in the liver, resulting in an improvement of glucose tolerance without any associated weight loss. ${ }^{8}$ This result indicates that restricting carbohydrate intake in the diet may improve the specific pathology of obese patients.

The $o b / o b$ mutant mice are characterized by obesity, hyperphagia, several metabolic abnormalities, such as glucose intolerance, hyperinsulinemia and hyperglycemia, which are based on the spontaneous mutation of the leptin gene. ${ }^{12}$ The ob/ob mice have rapidly increased their body weight until 3 months old, thereafter continues to gain slowly until 10 months of age. The blood glucose concentration also rose rapidly after weaning and reaches

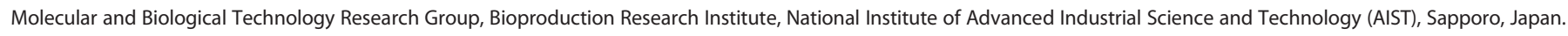

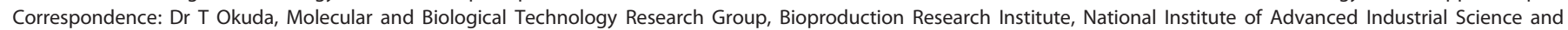
Technology (AIST), 2-17-2-1 Tsukisamu-Higashi, Toyohira-ku, Sapporo 062-8517, Japan. 
a peak between 2 and 3 months and then gradually decreases to a constant level by 4 to 5 months. ${ }^{13}$ Because the other pathological phenotypes such as hyperinsulinemia also developed in the juvenile stage (until 3 months old), we considered that the improvement of the hyperglycemic phenotype at this stage of ob/ ob mice should provide valid information about pathological processes of peripheral tissues induced by hyperglycemia.

In this study, we attempted to evaluate the effect of feeding KD on hyperglycemia by using ob/ob mice in the juvenile, rather than mature, stage. We established an animal model that has a chronically controlled glycemic status, which is established by being fed a regular or KD. This animal model was used to evaluate tissue pathologies induced by hyperglycemia at the molecular level. We found that feeding KD leads to an improved hyperglycemic and steatosis phenotype in juvenile ob/ob mice without any apparent health problems.

\section{MATERIALS AND METHODS}

\section{Animals and dietary studies}

All mice used in this study were female wild-type and ob/ob (B6.V-Lep $\left.{ }^{\mathrm{ob}} / \mathrm{J}\right)$ mice of the inbred strain C57BL/6J (Charles River Laboratories Japan Yokohama, Japan). Mice were maintained with a $12 \mathrm{~h}$ light/dark cycle in a temperature-controlled environment $\left(21 \pm 2^{\circ} \mathrm{C}\right)$. CE-2 (CLEA Japan, Tokyo, Japan) that comprised of $58.2 \%$ carbohydrate, $12.6 \%$ fat and $29.2 \%$ protein by calorie was used as regular chow. F3666 (Bio-Serv, Frenchtown, NJ, USA) that comprised of $1.7 \%$ carbohydrate, $93.9 \%$ fat and $4.4 \%$ protein by calorie was used as KD. Five-week-old mice were raised on either a chow or KD diet for a period of 7 weeks. During this period, the body weight and blood glucose level were monitored at the same time $(3: 00 \mathrm{pm})$ once a week. The Committee for the Experiments involving Animals of the National Institute of Advanced Industrial Science and Technology (AIST) approved all animal experiments.

\section{Blood chemical analysis}

Blood glucose levels were examined with venous blood collected from the tail vein by using the ACCU-CHEK Aviva meter system (Roche, Penzberg, Germany). Serum levels of $\beta$-hydroxybutyrate, a major ketone body produced in vivo, were measured as an indicator of the levels of ketone bodies with the Precision Xceed $\beta$-Ketone Monitoring System (Abbott Laboratories, Abbott Park, IL, USA). Serum insulin levels were measured with the Mouse Insulin ELISA (enzyme-linked immunosorbent assay) Kit High Range Speedy (Morinaga Institute of Biological Science, Yokohama, Japan). Total triglycerides, cholesterol and non-esterified fatty acid levels were measured with commercial enzyme colorimetric assay kits (Wako Pure Chemical Industries, Osaka, Japan). The triglycerides and cholesterol profiles in serum lipoproteins were determined by the high-sensitivity lipoprotein profiling system using high-performance liquid chromatography (Skylight Biotech Inc., Akita, Japan).

Total lipids in the liver were extracted with chloroform/methanol 2:1 $\left(v v^{-1}\right)$ as reported previously. ${ }^{14}$ The total lipids extracted from $100 \mathrm{mg}$ of liver were dissolved in the $1 \mathrm{ml}$ isopropanol, and the concentration of triglycerides and cholesterol were measured with enzyme colorimetric assay kits as described above. The amount of overall hepatic lipid was then calculated based on the total weight of the liver.

\section{Preparation of protein extracts}

Proteins were extracted from each isolated tissue ( $80 \mathrm{mg}$ of weight) into $0.4 \mathrm{ml}$ of $20 \mathrm{~mm}$ HEPES/0.25 $\mathrm{m}$ sucrose buffer ( $\mathrm{pH} 7.5$ ) supplemented with $0.1 \mathrm{~mm}$ phenylmethylsulfonyl fluoride and a proteinase inhibitor cocktail (Complete mini EDTA-free; Roche) by using a disposable homogenizer (Biomasher II; Nippi, Tokyo, Japan). After centrifugation of the homogenates at $800 \mathrm{~g}$ for $15 \mathrm{~min}$ at $4{ }^{\circ} \mathrm{C}$, the supernatants were filtered using a Ultrafree-MC $(0.45 \mu \mathrm{m})$ centrifugal device (Millipore, Billerica, MA, USA) at $13000 \mathrm{~g}$ for $3 \mathrm{~min}$ at $4{ }^{\circ} \mathrm{C}$. The filtrates were used as protein samples in subsequent experiments. The concentration of protein in the sample was determined by using the Bio-Rad Protein Assay (Bio-Rad Laboratories, Hercules, CA, USA).

\section{Protein analysis}

The proteins $(5 \mu \mathrm{g})$ were separated by sodium dodecyl sulfate-polyacrylamide gel electrophoresis using a polyacrylamide gel (SuperSep 5-20\% Gel; Wako, Osaka, Japan), and the gel was used for LC-MS/MS analysis or western blotting. For the LC-MS/MS analysis, the gel was stained with Coomassie Brilliant Blue and the relevant protein band was excised. After limited proteolysis of the proteins in the excised gel by trypsin, the ion mass spectra of the peptide products were determined by an LC-MS/MS analytical service performed by APRO Life Science Institute (Naruto, Japan). The determined ion mass spectra were sequentially searched in a peptide mass sequence database using the MASCOT algorithm (Mascot Server, http://www.matrixscience.com/server.html; Matrix Science, Boston, MA, USA) for protein identification. For western blotting, the protein bands from the gel were transferred onto Immobilon-P PVDF membranes (Millipore) by electroblotting at a constant current of $90 \mathrm{~mA}$ for $1 \mathrm{~h}$. After blotting, the membrane was incubated with the monoclonal anti-FAS antibody (C20G5) or the polyclonal anti-ACC1 antibody (Cell Signaling Technology, Danvers, MA, USA), or the monoclonal anti-actin antibody (mAb C4; BD Biosciences, Franklin Lakes, NJ, USA), respectively. The membranes were sequentially incubated with horse radish peroxidaselinked secondary antibody, and the antibody binding was then detected with ECL Plus Western Blotting Detection Reagents (GE Healthcare UK Ltd Amersham, UK) by using the image analyzer Light-Capture (ATTO, Tokyo, Japan).

\section{ELISA}

A measure of $20 \mu \mathrm{g} \mathrm{ml}^{-1}$ of protein samples $(50 \mu \mathrm{l})$ were applied onto a 96-well microtiter plate (Nunc MaxiSorp F96; Thermo Fisher Scientific, Waltham, MA, USA), and incubated overnight at $4{ }^{\circ} \mathrm{C}$. After wash two times with phosphate-buffered saline (PBS), $50 \mu$ l of blocking buffer (1\% bovine serum albumin in PBS) were added into wells and incubated for $15 \mathrm{~min}$ at room temperature, followed by adding primary antibodies into wells with appropriate dilution ratio. After $3 \mathrm{~h}$ incubation at room temperature, wells were washed three times with $0.1 \%$ Tween-20 in PBS, and then $0.1 \%$ Tween-20 in PBS-diluted horse radish peroxidase-linked secondary antibody was added. An horse radish peroxidase substrate (1-Step Uitra TMBELISA Substrate; Thermo Fisher Scientific) was used for the detection of antibody bindings, and the results were measured as absorbance at $450 \mathrm{~nm}$. Wells without primary antibody reaction were then used for monitoring of background absorbance caused by nonspecific bound of secondary antibody. Anti-FAS, anti-ACC1 and anti-actin antibodies were used as primary antibodies.

Fatty acid analysis

The fatty acid profiles in the total lipids of the liver and serum were determined as follows. Fatty acids were first converted to their methyl esters and then analyzed by gas-liquid chromatography (GLC) as reported previously. ${ }^{15}$ In brief, total lipids from $10 \mu \mathrm{g}$ of the liver or $10 \mu \mathrm{l}$ of the serum were directly methanolyzed in $10 \%\left(\mathrm{vv}^{-1}\right)$ acetyl chloride in methanol at $100^{\circ} \mathrm{C}$ for $3 \mathrm{~h}$. Fatty acid methyl esters extracted with $n$ hexane were concentrated and then subjected to GLC on a gas chromatograph (model GC-1700; Shimadzu, Kyoto, Japan) equipped with a flame ionization detector and a capillary column (BPX70, $0.2 \mu \mathrm{m}$ (inner diameter) $\times 50 \mathrm{~m}$; SGE, Austin, TX, USA). Helium was used as the carrier gas. The injector and the detector were maintained at $260{ }^{\circ} \mathrm{C}$, and the column temperature was programed to increase from 160 to $240^{\circ} \mathrm{C}$ at a rate of $4{ }^{\circ} \mathrm{C} \mathrm{min}-1$ and then maintained at $240{ }^{\circ} \mathrm{C}$ for $5 \mathrm{~min}$. Compounds detected by GLC analysis were identified by comparison of their retention times in GLC analysis with those of known standards. The amount of fatty acid in each sample was calculated by GC analysis from the peak area of their fatty acid methyl esters using a data processor (C-R8A; Shimadzu) by comparison to the corresponding area of an internal standard. The amount of each lipid was calculated as the sum of the amounts of the constituent fatty acids detected.

\section{Histological analysis}

Livers were fixed with $4 \%$ paraformaldehyde in PBS, sectioned after paraffin embedding or freezing and then stained with hematoxylin-eosin or oil red $\mathrm{O}$, respectively. These experiments were performed by Histo Science Laboratory Co. Ltd (Tokyo, Japan). 
Statistical analysis

The significance was examined with Student's $t$-test. Examinations for the statistical analysis were performed with $n=4-9$ mice, from two independent experiments.

\section{RESULTS}

Establishment of a mouse model that improved chronic hyperglycemia

As reported previously, ${ }^{13}$ juvenile stage ob/ob mice (5-12 weeks old) of the inbred strain C57BL/6J exhibited chronic hyperglycemia after being fed a diet of regular chow (Figure 1a). Compared with the chow-fed wild-type mice of the inbred strain C57BL/6J, the blood glucose levels of the chow-fed ob/ob mice were consistently higher during the experimental period. This chronic hyperglycemic phenotype of $o b / o b$ mice was improved by
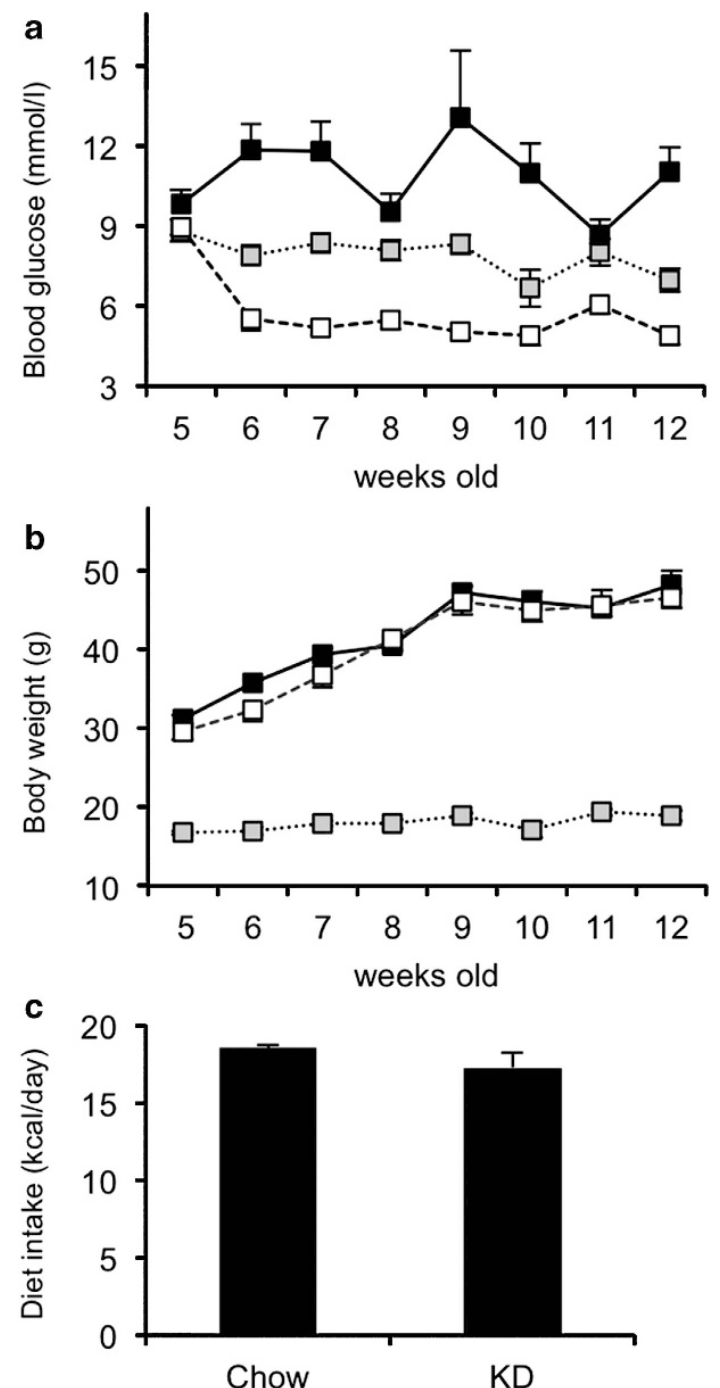

Figure 1. Effect of KD feeding on the blood glucose level and body weight of $o b / o b$ mice. Blood glucose levels (a) and body weights (b) of the chow- or KD-fed ob/ob mice during the experimental period (5-12 weeks of age). The parameters for chow-fed wild-type mice $(\mathrm{C} 57 \mathrm{BL} / 6 \mathrm{~J})$ are shown as a reference. Filled squares with solid line, chow-fed $o b / o b$ mice; open squares with dashed line, KD-fed $o b / o b$ mice; gray squares with dotted line, chow-fed wild-type mice. Means \pm s.e., $n=9$ from two independent experiments. (c) The average daily quantity (kcal) of diet intake in chow- or KD-fed ob/ob mice group during the experimental period. Means \pm s.e., $n=4$.
KD feeding. The KD-fed ob/ob mice always exhibited lower blood glucose levels than those of chow-fed wild-type mice. The average blood glucose level during the experimental period of each feeding model was as follows: $10.86 \mathrm{mmoll}^{-1}$ (chow-fed ob/ob mice), $5.75 \mathrm{mmoll}^{-1}$ (KD-fed ob/ob mice) and $7.90 \mathrm{mmoll}^{-1}$ (chow-fed wild-type mice).

By contrast, the KD feeding did not influence the body weight gain of $o b / o b$ mice (Figure $1 b$ ). Both the chow-fed $o b / o b$ mice and $\mathrm{KD}$-fed $o b / o b$ mice exhibited an obese phenotype, that is, in each case their body weight reached approximately $50 \mathrm{~g}$ by 12 weeks of age. Caloric intakes in both mice were also almost equivalent (Figure 1c). The averages of caloric intake of both mice during the experimental period were approximately $18 \mathrm{kcal}$ per day. At the end point of the feeding experiment, sera and tissue samples were collected from both chow- and KD-fed ob/ob mice, and used in the following analysis.

Improvement of hepatic steatosis phenotype in ob/ob mice by KD feeding

Next, we examined the morphology of several organs and tissues derived from each mouse. The progression to hepatic steatosis was observed in the liver of chow-fed ob/ob mice, but not in KDfed ob/ob mice (Figure 2a). No apparent morphological changes could be observed in the other organs and tissues. The average weight of the liver from KD-fed ob/ob mice was approximately $30 \%$ of that of the chow-fed ob/ob mice (Table 1). In the
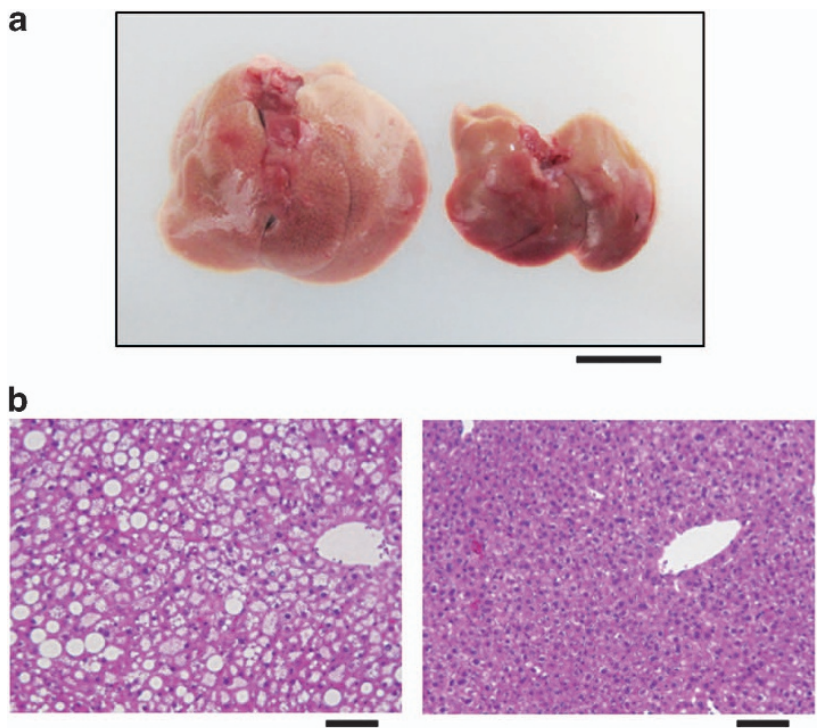

C
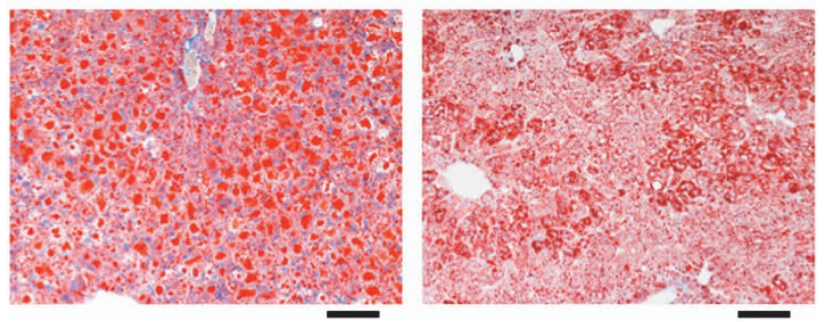

Figure 2. Histological analysis of the liver from ob/ob mice. (a) Representative morphology of the liver from chow-fed (left) and KDfed (right) $o b / o b$ mice. (b) Paraffin sections prepared from chow-fed (left panel) or KD-fed (right panel) ob/ob mice were stained with hematoxylin-eosin. (c) Cryosections prepared from chow-fed (left panel) or KD-fed (right panel) ob/ob mice were stained with oil red O. Scale bars indicate $10 \mathrm{~mm}$ (a) or $100 \mu \mathrm{m}$ (b and $\mathbf{c}$ ). 
histological analysis, a number of large vacuoles were confirmed in the liver sections of chow-fed ob/ob mice, which were not observed in the liver of KD-fed ob/ob mice (Figure 2b). These vacuoles were identified as large lipid-filled vacuoles by oil red $\mathrm{O}$ staining procedure (Figure 2c). Furthermore, biochemical analysis also supported these results. Specifically, a large amount of triglycerides were detected in the liver of chow-fed ob/ob mice, which was considerably higher than that found in the liver of KDfed ob/ob mice (Table 1).

Characterization of biochemical parameters in the serum of ob/ob mice

Serum biochemical analysis revealed that feeding KD influenced several biochemical parameters in the serum of ob/ob mice (Table 1$)$. In addition, the ketone body ( $\beta$-hydroxybutyrate) levels in the serum were increased in KD-fed $o b / o b$ mice. Indeed, the average level of ketone bodies in KD-fed ob/ob mice was approximately fivefold higher than that of the chow-fed $o b / o b$ mice. KD feeding improved hyperglycemia and also resulted in a decrease in the serum insulin levels of $o b / o b$ mice. Serum nonesterified fatty acids, triglycerides and cholesterol levels as a whole remained elevated in the KD-fed ob/ob mice. In particular, the cholesterol levels were significantly increased by feeding KD. Further analysis of lipoprotein fractions revealed that for KD-fed $o b / o b$ mice, the levels of triglycerides in the low-density lipoprotein (LDL) fraction and cholesterol in the high-density lipoprotein (HDL) fraction were increased, but both were significantly decreased in the chylomicron fraction. Owing to the increased plasma HDL phenotype in ob/ob mice, ${ }^{16,17}$ serum cholesterol was mainly found in the HDL fraction of $o b / o b$ mice fed with either the KD or the chow diet. A decrease in the

\begin{tabular}{|c|c|c|}
\hline Parameters & Chow & $K D$ \\
\hline \multicolumn{3}{|l|}{ Serum } \\
\hline $\begin{array}{l}\beta \text {-Hydroxybutyrate } \\
\left(\mathrm{mmoll}^{-1}\right)\end{array}$ & $0.27 \pm 0.03$ & $1.05 \pm 0.25^{*}$ \\
\hline Insulin (pmoll-1) & $4019.72 \pm 568.23$ & $217.54 \pm 7.93^{* *}$ \\
\hline NEFAs $\left(\mathrm{mmoll}^{-1}\right)$ & $0.98 \pm 0.17$ & $1.68 \pm 0.20^{* * *}$ \\
\hline \multicolumn{3}{|l|}{ Triglycerides (mmoll ${ }^{-1}$ ) } \\
\hline Total & $1.23 \pm 0.22$ & $1.47 \pm 0.30$ \\
\hline CM & $0.31 \pm 0.06$ & $0.08 \pm 0.02^{*}$ \\
\hline VLDL & $0.58 \pm 0.11$ & $0.53 \pm 0.14$ \\
\hline LDL & $0.23 \pm 0.04$ & $0.65 \pm 0.17^{* * *}$ \\
\hline $\mathrm{HDL}$ & $0.08 \pm 0.02$ & $0.07 \pm 0.02$ \\
\hline \multicolumn{3}{|l|}{ Cholesterol $\left(\mathrm{mmoll}^{-1}\right)$} \\
\hline Total & $4.08 \pm 0.28$ & $5.66 \pm 0.18^{* *}$ \\
\hline CM & $0.05 \pm 0.01$ & $0.01 \pm 0.001^{* *}$ \\
\hline VLDL & $0.16 \pm 0.03$ & $0.09 \pm 0.004^{* * *}$ \\
\hline LDL & $0.71 \pm 0.06$ & $0.81 \pm 0.04$ \\
\hline $\mathrm{HDL}$ & $3.18 \pm 0.24$ & $4.66 \pm 0.15^{* *}$ \\
\hline \multicolumn{3}{|l|}{ Liver } \\
\hline Weight (g) & $3.59 \pm 0.06$ & $1.11 \pm 0.07^{* *}$ \\
\hline Total triglyceride $(\mathrm{mg})$ & $361.93 \pm 6.01$ & $74.20 \pm 4.70^{* *}$ \\
\hline Total cholesterol (mg) & $38.16 \pm 0.63$ & $5.89 \pm 0.37^{* *}$ \\
\hline
\end{tabular}

Abbreviations: Chow, regular chow diet; $\mathrm{CM}$, chylomicron; HDL, highdensity lipoprotein; $K D$, ketogenic diet; $L D L$, low-density lipoprotein; NEFAs, non-esterified fatty acids; VLDL, very-low-density lipoprotein. Biochemical examinations were performed in $o b / o b$ mice after 7 weeks feeding on Chow or KD. Data are presented as means \pm s.e.; $n=9$ (serum) or $n=4$ (liver). ${ }^{*} P<0.01,{ }^{*} P<0.001$ and ${ }^{* *} P<0.05$, Chow vs KD. cholesterol level was also observed in the very-low-density lipoprotein (VLDL) fraction in the KD-fed ob/ob mice.

\section{Protein expression in the liver of $o b / o b$ mice after KD feeding} To clarify the molecular mechanism of the observed improvement in steatosis upon feeding KD, we examined protein expression in the liver extracts of chow-fed ob/ob mice, KD-fed ob/ob mice and chow-fed wild-type mice. Analysis of the extracts by sodium dodecyl sulfate-polyacrylamide gel electrophoresis combined with Coomassie Brilliant Blue staining revealed a striking difference in protein expression among these three groups of mice (Figure 3a). The protein expression pattern of KD-fed ob/ob mice (Figure 3a, lanes 6-10) was very similar to that of chow-fed wild-type mice (Figure $3 a$, lane 0 ). In contrast, accumulation of a distinctive, highmolecular-weight protein band was observed in the extract of chow-fed ob/ob mice (Figure 3a, lanes 1-5, arrow (X)). Next, we attempted to identify this upregulated protein, which we anticipated to be a key factor in promoting steatosis in chowfed ob/ob mice. These experiments were performed by LC-MS/MS analysis in conjunction with the MASCOT protein identification software as described in Materials and methods. The results are shown in Table 2. The band was composed of at least four proteins: FAS, ACC1, non-muscle heavy-chain myosin II-A and talin-1. We also confirmed these changes to the protein expression profile by western blotting (Figure $3 b$ ). This analysis

a
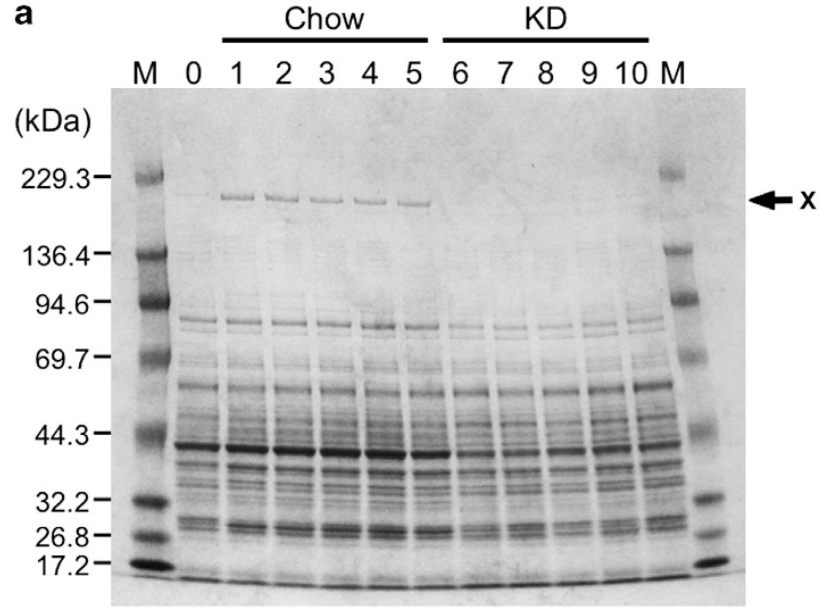

b Chow KD

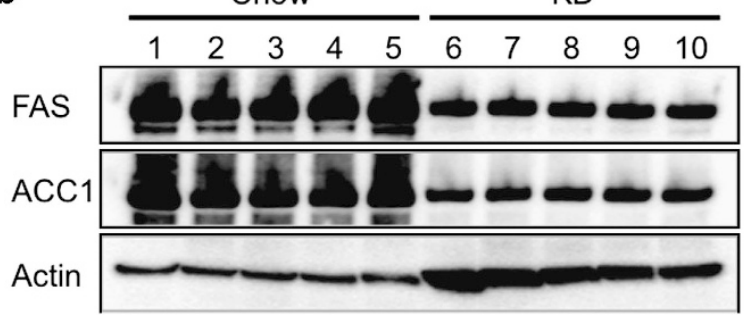

Figure 3. Protein analysis of liver extracts from $o b / o b$ mice. Individual liver extracts of chow-fed (lanes 1-5) and KD-fed ob/ob mice (lanes 6-10) were separated by sodium dodecyl sulfatepolyacrylamide gel electrophoresis, and stained with Coomassie Brilliant Blue (a) or analyzed by western blotting (b). (a) The arrow (X) highlights the accumulated protein band in chow-fed ob/ob mice (lanes 1-5). The liver extract of chow-fed wild-type mice (lane 0 ) was loaded as a reference. Lane M, size marker. (b) Protein expression of FAS (upper panel), ACC1 (middle panel) and actin (lower panel). 
Table 2. Proteins identified in X by LC-MS/MS with MASCOT database search

\begin{tabular}{lcccc}
\hline $\begin{array}{l}\text { Protein } \\
\text { identified }\end{array}$ & Score & $\begin{array}{c}\text { Nominal } \\
\text { mass }\end{array}$ & $\begin{array}{c}\text { Peptide } \\
\text { matched }\end{array}$ & $\begin{array}{c}\text { Accession } \\
\text { number }\end{array}$ \\
\hline FAS & 3672 & 272257 & 91 & gi|93102409 \\
ACC1 & 584 & 264953 & 11 & gi|125656173 \\
$\begin{array}{l}\text { Non-muscle } \\
\text { heavy-chain }\end{array}$ & 210 & 226217 & 4 & gi|17978023 \\
$\begin{array}{l}\text { myosin II-A } \\
\text { Talin-1 }\end{array}$ & 137 & 269665 & 3 & gi|227116327 \\
\hline
\end{tabular}

Abbreviations: ACC1, acetyl-CoA carboxylase 1; FAS, fatty acid synthase; LC-MS/MS, liquid chromatography with tandem mass spectrometry. A score $>56$ indicates statistical significance $(P<0.05)$ by the MASCOT algorithm. 'Peptide matched' represents the number of matched peptide sequences in the identified protein, which were detected by LC-MS/MS analysis. The 'Accession number' indicates the number of identified proteins in the NCBInr database (http://www.ncbi.nlm.nih.gov/).

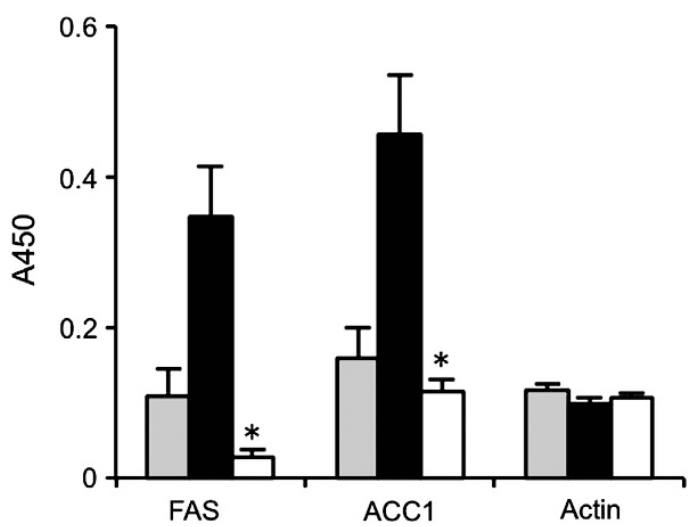

Figure 4. The expression levels of FAS, ACC1 and actin were measured by ELISA. Individual protein extracts of the liver in chow-fed (closed bars) or KD-fed (open bars) ob/ob mice were analyzed by ELISA. Protein extracts prepared from the liver of chowfed wild-type mice were indicated as a reference (gray bars). Means \pm s.d., $n=9$ from two independent experiments. ${ }^{*} P<0.001$ chow-fed vs KD-fed ob/ob mice.

established that the protein band was primarily made up of FAS and ACC1 (Figure 3b, upper and middle panels). The other proteins were undetectable by western blotting (data not shown). By comparison to the liver extracts of the chow-fed ob/ob mice, the expression levels of FAS and ACC1 in KD-fed ob/ob mice were drastically reduced. The ELISA analysis revealed that the relative amount of these proteins in the liver extracts of KD-fed ob/ob mice were approximately $92 \%$ (FAS) or $75 \%$ (ACC1) lower than that of chow-fed ob/ob mice (Figure 4). In contrast, there was no significant difference between the two groups in the expression levels of actin, an internal control in the protein extract. These results indicate that a large amount of FAS and ACC1 (and other unidentified proteins) accumulate in the liver of chow-fed $o b / o b$ mice, but not in ob/ob mice fed KD.

Fatty acid composition of the liver and serum of ob/ob mice In the liver, excess glucose is metabolized to acetyl-CoA and sequentially converted to fatty acids via the de novo lipogenesis pathway. Triglycerides synthesized from the fatty acids are either stored in the hepatic lipid-filled vacuoles or secreted from the liver in the form of VLDL. ${ }^{18}$ Because FAS and ACC1 are known as the central enzymes of lipogenesis in the liver, ${ }^{19}$ it was expected that
Table 3. Fatty acid composition in the liver and serum of ob/ob mice

\begin{tabular}{|c|c|c|c|c|c|c|}
\hline \multirow[t]{2}{*}{ Species } & \multicolumn{2}{|c|}{ Diet } & \multicolumn{2}{|c|}{ Liver } & \multicolumn{2}{|c|}{ Serum } \\
\hline & Chow & $K D$ & Chow & $K D$ & Chow & $K D$ \\
\hline C14:0 & 0.6 & 2.9 & 0.6 & 0.4 & TR & $\mathrm{TR}$ \\
\hline C16:0 & 16.9 & 22.7 & 23.1 & 22.6 & 21.6 & 16.3 \\
\hline C16:1 & 1.3 & 1.5 & 9.5 & 1.1 & 3.8 & 0.4 \\
\hline C18:0 & 2.2 & 11.2 & 2.8 & 8.3 & 11.2 & 15.5 \\
\hline C18:1 & 23.9 & 35.3 & 52.5 & 23.9 & 23.0 & 9.0 \\
\hline C18:2 & 45.2 & 22.5 & 6.8 & 30.6 & 20.7 & 33.4 \\
\hline C18:3 & 2.9 & 0.9 & ND & 0.9 & TR & TR \\
\hline C20:1 & ND & ND & 0.7 & 0.4 & TR & TR \\
\hline C20:3 & ND & ND & 0.4 & 0.8 & 1.0 & 0.6 \\
\hline C20:4 & ND & ND & 1.0 & 4.9 & 6.9 & 14.8 \\
\hline C20:5 & 1.5 & ND & ND & ND & 4.1 & 0.9 \\
\hline C22:5 & ND & ND & 0.2 & 0.5 & 0.5 & 0.9 \\
\hline C22:6 & ND & ND & 0.8 & 3.0 & 3.5 & 2.5 \\
\hline Others & 5.5 & 3.0 & 1.6 & 2.6 & 3.7 & 5.7 \\
\hline
\end{tabular}

Abbreviations: Chow, regular chow diet; GLC, gas-liquid chromatography; $\mathrm{KD}$, ketogenic diet; ob, obese. Fatty acid analysis was conducted by GLC. Data represent ratio of each fatty acid species to total fatty acids (\%) prepared from the liver and serum of Chow- or KD-fed ob/ob mice, respectively. The column of Diet indicates fatty acid profiles of Chow (CE-2) and KD (F3666) using this study. Values indicate ND, not detected; TR, trace.

altered expression of these enzymes should elicit changes in the structural composition of fatty acids in this tissue. GLC analysis showed that C18:1 (oleic acid or vaccenic acid), a major product in the liver, ${ }^{20,21}$ was detected as the majority of total fatty acid in the liver of chow-fed ob/ob mice (Table 3 ). In contrast, the ratio of C18:1 was decreased by feeding KD. In the liver of KD-fed ob/ob mice, C18:2 (linoleic acid) was primarily detected among fatty acid species, and the ratio of C20:4 (arachidonic acid), which is synthesized by linoleic acid in mammalian tissues, especially the liver, ${ }^{22}$ was also increased. The lipids from the liver are released to circulate in the bloodstream. Therefore, we considered that the change in the structural composition of the fatty acids might be reflected by the fatty acids in the peripheral blood. To confirm this hypothesis, we analyzed the fatty acids in the serum of $o b / o b$ mice. As expected, C18:1 was the main fatty acid in the serum of chow-fed mice, whereas C18:2 was the main fatty acid in the serum of KD-fed mice. However, both C18:2 and C20:5 (eicosapentaenoic acid) had a low or undetectable content in the liver of chow-fed ob/ob mice, but were readily detected in the serum. Because the chow (CE-2) contains a large amount of C18:2 and a smaller amount of $\mathrm{C} 20: 5$, the source of these fatty acids in the serum is presumably from the intake of CE-2. By contrast, despite the high content of this fatty acid in the serum of the two groups of mice (chow- and KD-fed ob/ob mice), C20:4 was undetectable in both diets. This result indicates that the C20:4 found in the serum is a biosynthetic product of the mouse tissues.

\section{DISCUSSION}

In this study, we established a mouse model that improves the hyperglycemic phenotype of $o b / o b$ mice by feeding KD beginning at juvenile stage. In this model, we also succeeded in preventing the progression of hepatic steatosis by feeding KD, which involves long-term suppression of hyperglycemia. Nonetheless, both the chow- and KD-fed ob/ob mice underwent considerable weight gain and exhibited an obese phenotype. A previous study using mature ob/ob mice whose body weights reached to $45 \mathrm{~g}$ also reported that their weight gain was unaffected by long-term KD feeding. ${ }^{8}$ This finding indicates that the peripheral tissues in the $o b / o b$ mice were supplied with sufficient quantities of energy nutrients to allow weight gain regardless of whether they were fed 
KD or chow. In fact, we found that feeding KD helped in maintaining a constant level of blood glucose in addition to high levels of ketone bodies (Figure 1a and Table 1). These energy nutrients are produced by $\beta$-oxidation or the gluconeogenesis pathway in vivo under KD feeding.

The drastic suppression in the hepatic steatosis phenotype of $\mathrm{KD}$-fed $o b / o b$ mice was not observed in KD-fed ob/ob mouse after reaching their body weight of $45 \mathrm{~g}$ as reported previously. ${ }^{8}$ Because this mature ob/ob mice have already been shown to display a steatosis phenotype, we reasoned that it should be possible to evaluate the effect of KD feeding on the liver by using juvenile $o b / o b$ mice. KD feeding could not improve the steatosis phenotype in $\mathrm{C} 57 \mathrm{BL} / 6 \mathrm{~J}$ mice, rather aggravated in some other reports. $8,9,23,24$ These results suggest that the improvement of hepatic steatosis in ob/ob mice is a unique phenotype observed only during their juvenile period. Our experiments subsequently revealed the preventive effect of this diet on the development of steatosis in juvenile ob/ob mice. Analysis of protein expression in the liver supports this result; specifically, we found an excessive accumulation of FAS and ACC1 in the fatty liver of chow-fed ob/ob mice (Figures 3 and 4 and Table 2). FAS and ACC1 play a pivotal role in de novo lipogenesis, which produces fatty acids from surplus glucose. ${ }^{19}$ Excessive intake of nutrients induces hyperglycemia, with an increase in blood insulin and promotion of lipogenesis in the liver. Several fatty acids (especially C18:1) are endogenously synthesized, which are likely to serve as major substrates for hepatic triglyceride synthesis. Indeed, this is the principal factor in the development of steatosis. ${ }^{18}$ Although $\mathrm{C} 18: 1$ is not the major component of chow (CE-2), it was found in the majority of the liver fatty acids from chow-fed ob/ob mice (Table 3 ). This finding indicates that accumulation of C18:1 in the liver of chow-fed $o b / o b$ mice is related to lipogenesis. Stearoyl-CoA desaturase 1 is a key enzyme for the lipogenesis of C18:1 and has been proposed to regulate the rate of lipogenesis and activate the $\beta$-oxidation pathway. ${ }^{19}$ Several studies have demonstrated that the suppression of stearoyl-CoA desaturase 1 gene expression prevents the development of steatosis by inhibition of de novo lipogenesis and activation of the $\beta$-oxidation pathway. ${ }^{25,26}$ The suppressive effect of $\mathrm{KD}$ on the gene expression of stearoyl-CoA desaturase 1 as well as FAS has been reported in several established KD-fed mice models. ${ }^{8,9}$ Thus, it is reasonable to believe that the reduction of de novo lipogenesis in KD-fed juvenile $o b / o b$ mice contributed to inhibit the development of steatosis. Although it is reported that the decrease of the transcript level of FAS is observed in KD-fed mature ob/ob mice, any improvement of steatosis phenotype is not observed in the liver. ${ }^{8}$ From these results, it is difficult to explain the improvement of steatosis phenotype only from decreased levels of FAS and ACC1 involving suppression of lipogenesis by KD feeding. In this study, we used $o b / o b$ mice at the stage of rapid progression of obesity, during which several drastic changes such as augmentation of insulin secretion occur. Thus, it is suggested that not only the reduction of de novo lipogenesis by KD feeding but also some other factors that are specific to this stage of $o b / o b$ mice will be necessary to improve the steatosis phenotype. Further investigation will be needed to find these factors, which is expected to provide useful information to develop new medical treatments for steatosis.

The molecular basis for the upregulation of genes involved in lipogenesis has been well characterized. ${ }^{19}$ Under conditions of hyperglycemia associated with increase serum insulin, genes encoding lipogenic enzymes are upregulated in the liver by the action of transcriptional factors such as SREBP-1c, ChREBP and liver $X$ receptors. ${ }^{19,27-29}$ In addition, the mechanisms by which the genes encoding FAS and ACC1 are induced by these transcriptional factors have also been elucidated. ${ }^{30-34}$ From these observations, we conclude that the improvement in hyperglycemia by KD feeding prevented the development of steatosis in juvenile ob/ob mice. Moreover, we also suggest that intake of excessive glucose rather than fat has an important role in the progression of steatosis.

We propose that the decreased expression of lipogenic enzymes after feeding KD also induced changes in the fatty acid composition observed in KD-fed ob/ob mice. GLC analysis revealed that C18:1, the major product of hepatic lipogenesis, is the main component of total fatty acids in the fatty liver of chow-fed ob/ob mice, whereas the ratio of this fatty acid was lower in the liver of KD-fed ob/ob mice (Table 3). Although C18:1 is a major component of KD, the content of this fatty acid in KD-fed ob/ob mice liver is lower than that of C18:2. The reduction in the level of C18:1 infers the decreased endogenous synthesis of fatty acid due to decreased FAS and ACC1, and also stearoyl-CoA desaturase 1 expression. In the KD-fed ob/ob mice, C18:2 was found to be a major component of total fatty acids in the liver and serum. It is well known that C18:2 is an essential fatty acid and cannot be synthesized in mammalian cells. The KD used in these experiments contained C18:2. Thus, C18:2 found in the liver of KD-fed $o b / o b$ mice is mostly derived from dietary intake. An increase in the amount of C18:2 in the liver and serum of KD-fed ob/ob mice was coincident with an increase in the level of C20:4, which is synthesized from C18:2 in mammalian tissues, especially liver. ${ }^{22}$ Importantly, there was also an increase in the amount of dietaryderived fatty acids, such as C20:5, in the serum but not in the liver of chow-fed ob/ob mice. These compositional changes in the fatty acid profile of the serum might reflect the pathological condition of the liver. Furthermore, these factors are expected to provide novel diagnostic indices for the evaluation of the pathological condition resulting from alterations to the diet.

The distribution pattern of serum triglycerides in the lipoprotein fraction from KD- and chow-fed ob/ob mice was complex (Table 1). We found lower levels of serum triglycerides and cholesterol in the chylomicron fraction of KD-fed ob/ob mice, indicating a decrease of serum chylomicrons by KD feeding. We consider that serum chylomicrons are rapidly absorbed in the liver of KD-fed $o b / o b$ mice, but poorly absorbed in the fatty liver of chow-fed $o b / o b$ mice. In contrast, higher levels of triglycerides were found in the LDL fraction from KD-fed ob/ob mice. This result infers that triglycerides stored in the liver of KD-fed $o b / o b$ mice are readily released into the serum. In this model, the levels of triglycerides and cholesterol in the serum of KD-fed ob/ob mice were relatively high in comparison to the serum of chow-fed ob/ob mice. Conversely, the levels of triglycerides and cholesterol were markedly lower in the liver of KD-fed $o b / o b$ mice than in the liver of chow-fed $o b / o b$ mice. This result indicates that the progression of steatosis was specifically blocked in KD-fed ob/ob mice, in spite of their obese phenotype and high levels of triglyceride in the serum. Investigation of these unique phenotypes is expected to provide valuable information concerning the development of steatosis and possible new therapies to ameliorate the condition.

In conclusion, our established mouse model demonstrated the effectiveness of a KD for improving hyperglycemia and thereby preventing the development of steatosis. We propose that the established animal model will be a useful tool for understanding the pathologies induced by hyperglycemia.

\section{CONFLICT OF INTEREST}

The authors declare no conflict of interest.

\section{ACKNOWLEDGEMENTS}

This work was supported by JSPS KAKENHI (22700780 and 24700859) and by the Sasakawa Scientific Research Grant from The Japan Science Society. 


\section{REFERENCES}

1 Kilpatrick ES, Rigby AS, Atkin SL. The Diabetes Control and Complications Trial: the gift that keeps giving. Nat Rev Endocrinol 2009; 5: 537-545.

2 Stolar M. Glycemic control and complications in type 2 diabetes mellitus. Am J Med 2010; 123: S3-S11.

3 Singleton JR, Smith AG, Russell JW, Feldman EL. Microvascular complications of impaired glucose tolerance. Diabetes 2003; 52: 2867-2873.

4 Capes SE, Hunt D, Malmberg K, Gerstein HC. Stress hyperglycaemia and increased risk of death after myocardial infarction in patients with and without diabetes: a systematic overview. Lancet 2000; 355: 773-778.

5 Pannala R, Basu A, Petersen GM, Chari ST. New-onset diabetes: a potential clue to the early diagnosis of pancreatic cancer. Lancet Oncol 2009; 10: 88-95.

6 Messier C, Gagnon M. Glucose regulation and cognitive functions: relation to Alzheimer's disease and diabetes. Behav Brain Res 1996; 75: 1-11.

7 Yang X, Ongusaha PP, Miles PD, Havstad JC, Zhang F, So WV et al. Phosphoinositide signaling links O-GlcNAc transferase to insulin resistance. Nature 2008; 451: 964-969.

8 Badman MK, Kennedy AR, Adams AC, Pissios P, Maratos-Flier E. A very low carbohydrate ketogenic diet improves glucose tolerance in ob/ob mice independent of weight loss. Am J Physiol Endocrinol Metab 2009; 297: E1197-E1204.

9 Kennedy AR, Pissios P, Otu H, Roberson R, Xue B, Asakura K et al. A high-fat, ketogenic diet induces a unique metabolic state in mice. Am J Physiol Endocrinol Metab 2007; 292: E1724-E1739.

10 Poplawski MM, Mastaitis JW, Isoda F, Grosjean F, Zheng F, Mobbs CV. Reversal of diabetic nephropathy by a ketogenic diet. PLoS One 2011; 6: e18604.

11 Krebs P, Fan W, Chen YH, Tobita K, Downes MR, Wood MR et al. Lethal mitochondrial cardiomyopathy in a hypomorphic Med30 mouse mutant is ameliorated by ketogenic diet. Proc Natl Acad Sci USA 2011; 108: 19678-19682.

12 Zhang Y, Proenca R, Maffei M, Barone M, Leopold L, Friedman JM. Positional cloning of the mouse obese gene and its human homologue. Nature 1994; 372: 425-432.

13 Coleman DL, Hummel KP. The influence of genetic background on the expression of the obese $(O b)$ gene in the mouse. Diabetologia 1973; 9: 287-293.

14 Okuda T, Nakakita S, Nakayama K. Structural characterization and dynamics of globotetraosylceramide in vascular endothelial cells under TNF-alpha stimulation. Glycoconj J 2010; 27: 287-296.

15 Morita N, Nishida T, Tanaka M, Yano Y, Okuyama H. Enhancement of polyunsaturated fatty acid production by cerulenin treatment in polyunsaturated fatty acid-producing bacteria. Biotechnol Lett 2005; 27: 389-393.

16 Nishina PM, Lowe S, Wang J, Paigen B. Characterization of plasma lipids in genetically obese mice: the mutants obese, diabetes, fat, tubby, and lethal yellow. Metabolism 1994; 43: 549-553.

17 Silver DL, Jiang XC, Tall AR. Increased high density lipoprotein (HDL), defective hepatic catabolism of ApoA-I and ApoA-II, and decreased ApoA-I mRNA in ob/ob mice. Possible role of leptin in stimulation of HDL turnover. J Biol Chem 1999; 274: 4140-4146.

18 Miyazaki M, Kim YC, Gray-Keller MP, Attie AD, Ntambi JM. The biosynthesis of hepatic cholesterol esters and triglycerides is impaired in mice with a disruption of the gene for stearoyl-CoA desaturase 1. J Biol Chem 2000; 275: 30132-30138.

19 Postic C, Girard J. The role of the lipogenic pathway in the development of hepatic steatosis. Diabetes Metab 2008; 34: 643-648.
20 Jeffcoat R. Obesity - A perspective based on the biochemical interrelationship of lipids and carbohydrates. Med Hypotheses 2007; 68: 1159-1171.

21 Kasturi R, Joshi VC. Hormonal regulation of stearoyl coenzyme A desaturase activity and lipogenesis during adipose conversion of 3T3-L1 cells. J Biol Chem 1982; 257: 12224-12230.

22 Simopoulos AP. The importance of the omega-6/omega-3 fatty acid ratio in cardiovascular disease and other chronic diseases. Exp Biol Med 2008; 233: 674-688.

23 Jornayvaz FR, Jurczak MJ, Lee HY, Birkenfeld AL, Frederick DW, Zhang D et al. A high-fat, ketogenic diet causes hepatic insulin resistance in mice, despite increasing energy expenditure and preventing weight gain. Am J Physiol Endocrinol Metab 2010; 299: E808-E815.

24 Garbow JR, Doherty JM, Schugar RC, Travers S, Weber ML, Wentz AE et al. Hepatic steatosis, inflammation, and ER stress in mice maintained long term on a very low-carbohydrate ketogenic diet. Am J Physiol Gastrointest Liver Physiol 2011; 300: G956-G967.

25 Cohen P, Miyazaki M, Socci ND, Hagge-Greenberg A, Liedtke W, Soukas AA et al. Role for stearoyl-CoA desaturase-1 in leptin-mediated weight loss. Science 2002; 297: 240-243.

26 Ntambi JM, Miyazaki M, Stoehr JP, Lan H, Kendziorski CM, Yandell BS et al. Loss of stearoyl-CoA desaturase-1 function protects mice against adiposity. Proc Natl Acad Sci USA 2002; 99: 11482-11486.

27 Foretz M, Guichard C, Ferré P, Foufelle F. Sterol regulatory element binding protein-1c is a major mediator of insulin action on the hepatic expression of glucokinase and lipogenesis-related genes. Proc Natl Acad Sci USA 1999; 96: 12737-12742.

28 Yamashita H, Takenoshita M, Sakurai M, Bruick RK, Henzel WJ, Shillinglaw W et al. A glucose-responsive transcription factor that regulates carbohydrate metabolism in the liver. Proc Natl Acad Sci USA 2001; 98: 9116-9121.

29 Mitro N, Mak PA, Vargas L, Godio C, Hampton E, Molteni V et al. The nuclear receptor LXR is a glucose sensor. Nature 2007; 445: 219-223.

30 Bennett MK, Lopez JM, Sanchez HB, Osborne TF. Sterol regulation of fatty acid synthase promoter. coordinate feedback regulation of two major lipid pathways. $J$ Biol Chem 1995; 270: 25578-25583.

31 Lopez JM, Bennett MK, Sanchez HB, Rosenfeld JM, Osborne TF. Sterol regulation of acetyl coenzyme A carboxylase: a mechanism for coordinate control of cellular lipid. Proc Natl Acad Sci USA 1996; 93: 1049-1053.

$32 \mathrm{Kim}$ JB, Spiegelman BM. ADD1/SREBP1 promotes adipocyte differentiation and gene expression linked to fatty acid metabolism. Genes Dev 1996; 10: 1096-1107.

33 Dentin R, Pégorier JP, Benhamed F, Foufelle F, Ferré P, Fauveau V et al. Hepatic glucokinase is required for the synergistic action of ChREBP and SREBP-1C on glycolytic and lipogenic gene expression. J Biol Chem 2004; 279: 20314-20326.

34 Joseph SB, Laffitte BA, Patel PH, Watson MA, Matsukuma KE, Walczak R et al. Direct and indirect mechanisms for regulation of fatty acid synthase gene expression by liver X receptors. J Biol Chem 2002; 277: 11019-11025.

\section{(c)}

This work is licensed under the Creative Commons AttributionSOMERIGHISRESERVED NonCommercial-Share Alike 3.0 Unported License. To view a copy of this license, visit http://creativecommons.org/licenses/by-nc-sa/3.0/ 